\title{
Prenatal diagnosis of glutaric acidemia type 2 with the use of exome sequencing - an up-to-date review and new case report
}

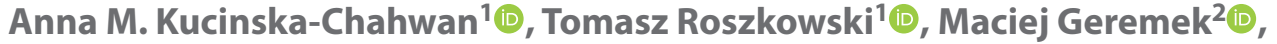

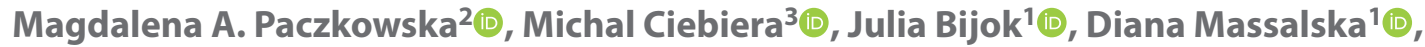 \\ Grzegorz Panek ${ }^{1}$, Krzysztof Siemion $^{4}{ }^{(0)}$, Beata A. Nowakowska² \\ ${ }^{1}$ Department of Gynecology Oncology and Obstetrics, Centre of Postgraduate Medical Education, Warsaw, Poland \\ ${ }^{2}$ Department of Medical Genetics, Institute of Mother and Child, Warsaw, Poland \\ ${ }^{3}$ Second Department of Obstetrics and Gynecology, Centre of Postgraduate Medical Education, Warsaw, Poland \\ ${ }^{4}$ Department of Pathology, Professor Witold Orlowski Public Clinical Hospital, \\ Medical Center for Postgraduate Education, Warsaw, Poland
}

\begin{abstract}
Introduction: Inborn errors of metabolism (IEM) also called metabolic diseases constitute a large and heterogenous group of disorders characterized by a failure of essential cellular functions. Antenatal manifestation of IEM is absent or nonspecific, which makes prenatal diagnosis challenging. Glutaric acidemia type $2(G A 2)$ is a rare metabolic disease clinically manifested in three different ways: neonatal-onset with congenital anomalies, neonatal-onset without congenital anomalies and late-onset. Neonatal forms are usually lethal. Congenital anomalies present on prenatal ultrasound as large, hyperechoic or cystic kidneys with reduced amniotic fluid volume.

Material and methods: We present a systematic literature review describing prenatal diagnosis of GA2 and a new prenatal case. Results: Ten prenatally diagnosed cases of GA2 have been published to date, mainly based on biochemical methods. New case of GA2 was diagnosed using exome sequencing method.

Discussion: All prenatal cases from literature review had positive history of GA2 running in the family. In our study trio exome sequencing was performed in case of fetal hyperechoic kidneys without a history of GA2. Consequently, we were able to identify two novel pathogenic variants of the ETFDH gene and to indicate their parental origin.

Summary: Exome sequencing approach used in case of fetal hyperechoic kidneys allows to identify pathogenic variants without earlier knowledge of the precise genetic background of the disease. Hyperechoic, enlarged kidneys could be one of the clinical features of metabolic diseases. After exclusion of chromosomal abnormalities, urinary tract obstruction and intrauterine infections, glutaric acidemia type 2 and number of monogenic disorders should be consider.
\end{abstract}

Key words: inborn errors of metabolism; glutaric acidemia type 2; multiple acyl-coenzyme A dehydrogenase deficiency; fetal abnormalities; prenatal diagnosis; exome sequencing

Ginekologia Polska 2021; 92, 1:51-56

\section{INTRODUCTION}

Inborn errors of metabolism - overview

Inborn errors of metabolism (IEM) also called metabolic diseases constitute a large and heterogenous group of disorders characterized by a failure of essential cellular functions. In the vast majority of IEM single gene mutation causes production of defective enzyme and in consequence disruption of cellular metabolic pathways leading to a deficiency of vital metabolites, deficiency of energy or accumulation of toxic substrates [1]. However, depending on the type of the mutation and its position along the gene residual enzyme activity in affected fetus is possible [2, 3]. Moreover, during pregnancy gas exchange, nutrients supply and metabolic waste elimination occur mainly due to maternal metabolism [4]. For these reasons many of IEM are asymptomatic in the fetus. Clinical features of IEM like vomiting, impaired multiorgan function, encephalopathy, hypoglycemia, hyperammonemia or acidemia starts days, weeks, months or even years after birth and are often life-threatening. However, in some of IEM severe metabolic disorder disrupt fetal 
development and congenital anomalies occur. Antenatal manifestation of IEM is nonspecific and include nonimmune hydrops fetalis, intrauterine growth restriction, central nervous system anomalies, heart defects, hyperechoic kidneys or skeletal anomalies what makes prenatal diagnosis of IEM challenging [5].

\section{Glutaric acidemia type 2 - basic facts}

One of the IEM associated with fetal anomalies is glutaric acidemia type 2 (GA2; OMIM \#231680). GA2 is a rare metabolic disease with a birth prevalence estimated at 1:250.000 [6]. Disease causing Multiple acyl-coenzyme A Dehydrogenase Deficiency (MADD) is clinically manifested in three different ways: neonatal-onset with congenital anomalies, neonatal-onset without congenital anomalies and late-onset. Both neonatal forms are usually lethal due to metabolic acidosis, hypoglycemia and multiorgan failure while late-onset form is highly variable and severe clinical deterioration or sudden death may occur at any age even without previous symptoms [3, 7]. In cases with prenatal manifestation congenital anomalies present on ultrasound as large, hyperechoic or cystic kidneys with reduced amniotic fluid volume [8]. Prenatal diagnosis of GA2 can be established based on dehydrogenase activity measurements, organic acids profile, acylcarnitine profile or genetic testing [9]. Pathogenic variants can be identified in one of the three genes (ETFA, ETFB, ETFDH) inherited in an autosomal recessive manner [10-12]. Differential diagnosis in cases with prenatally detected anomalies includes trisomy 13 or 18 , autosomal recessive polycystic kidney disease (ARPKD), renal cysts and diabetes syndrome, nephronophthisis, Joubert syndrome, Bardet-Biedl syndrome, Meckel-Gruber syndrome, oral-facial-digital syndrome type 1 and other rare monogenic disorders, cytomegalovirus intrauterine infection or urinary tract obstruction with kidney dysplasia [13]. Herein we present a systematic literature review of all prenatally detected cases of GA2. We also report on a first prenatal diagnosis of GA2 established by exome sequencing (ES) as an example of a diagnostic pathway from nonspecific sonographic features to exact genetic diagnosis.

\section{MATERIALS AND METHODS}

\section{Search strategy}

The authors performed a systematic literature review for any study reporting prenatal diagnosis of GA2 published between first description of the disease in 1976 until December 2019. Review was conducted using Pubmed/MEDLINE and Web of Science databases. The search strategy with following formula was applied: (glutaric aciduria OR glutaric acidemia OR madd) AND (prenatal OR antenatal OR fetus OR fetal). There was no language restriction placed on the manuscript search. Additionally, the references of

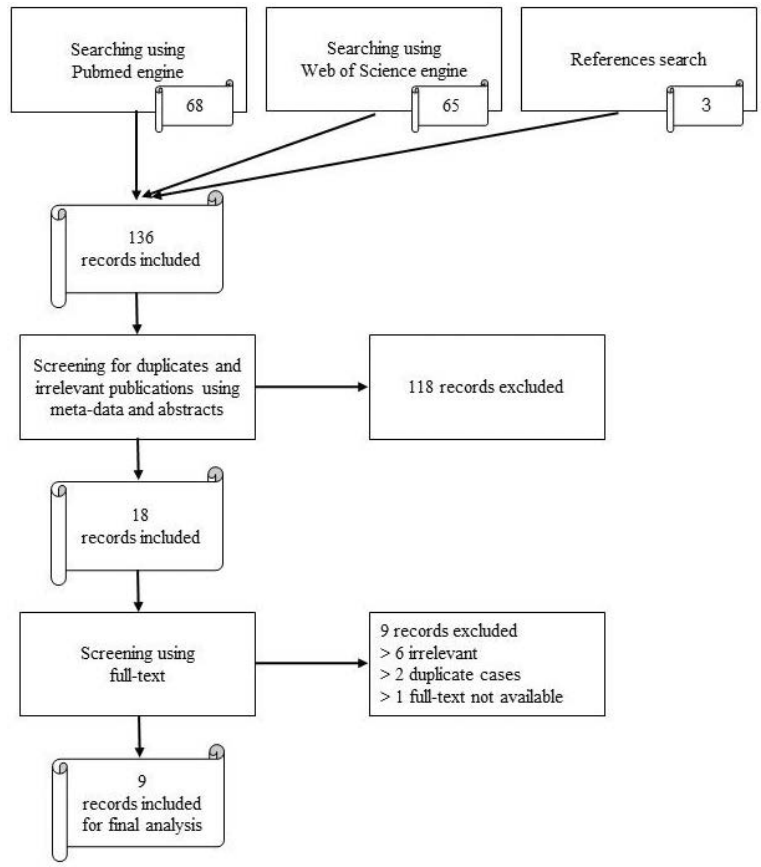

Figure 1. Flowchart for literature review

all selected manuscripts were screened for subsequent reports. Papers available in full text in which authors described methods used for prenatal diagnosis of GA2 in ongoing pregnancy not in stored material and in which diagnosis was positive were included. Papers not containing prenatal cases of GA2 or containing cases with negative diagnosis true or false were excluded. The systematic review flowchart and search strategy are summarized in Figure 1.

\section{New case report \\ Clinical presentation}

A 35-year-old patient presented in our ultrasound department for a detailed anomaly scan at 20 weeks of her third gestation due to history of complications in a previous pregnancies. Her first pregnancy ended in early miscarriage. Her second pregnancy was complicated by fetal anomalies - enlarged cystic kidneys, oligohydramnios and large cavum septi pellucidi (CSP). A full-term newborn delivered via cesarean section due to transverse presentation died 2 hours after birth. Prenatal karyotype was normal, and the patient was informed that a potential cause of fetal malformations was an autosomal recessive polycystic kidney disease (ARPKD) caused by PKHD1 gene mutation. However, an autopsy was not performed, and only a buccal swab was taken from the newborn for molecular tests. Sequencing for the most frequent pathogenic variants in exons 32 and 36 of PKHD1 gene gave negative result and ARPKD could not be confirmed nor excluded. The initial ultrasound scan at 20 weeks of patient's third pregnancy evaluated in our ultra- 
sound department revealed large CSP, enlarged hyperechoic kidneys and normal amount of the amniotic fluid (Fig. 2A). After a detailed ultrasound scanning, fetal urinary tract obstruction was excluded. Routine screening tests for rubella, toxoplasmosis, cytomegalovirus, hepatitis-B, hepatitis- $C$, HIV and syphilis were negative. After genetic counsel, due to positive history and current fetal anomalies, the patient decided to continue the pregnancy aware of high risk of fetal or neonatal death. Amniocentesis was performed for cytogenetic and molecular tests as described in next sections. During the third trimester amniotic fluid volume decreased leading to a Potter sequence (flattened nose, retrognathia, low-set abnormal ears, pulmonary hypoplasia, club feet caused by small amount of amniotic fluid surrounding the fetus). The child was liveborn at 37 weeks of pregnancy via cesarean section due to breach presentation and died after 2 hours from metabolic and multiorgan failure. In the postmortem examination both kidneys were enlarged. In cross section no cyst has been found during the macroscopic examination (Fig. 2B). The lungs appeared hypoplastic. The liver seemed to be enlarged, but no pathologic findings on cross section were stated. No abnormalities were found in the heart, central nervous system, bile and pancreatic ducts. On microscopic examination both kidneys contained many round, simple cysts, which were lined by cuboidal or flattened epithelium. In the medulla they were surrounded by loose mature mesenchymal tissue (Fig. 2C-D). The liver presented features of adiposis and preserved extramedul-

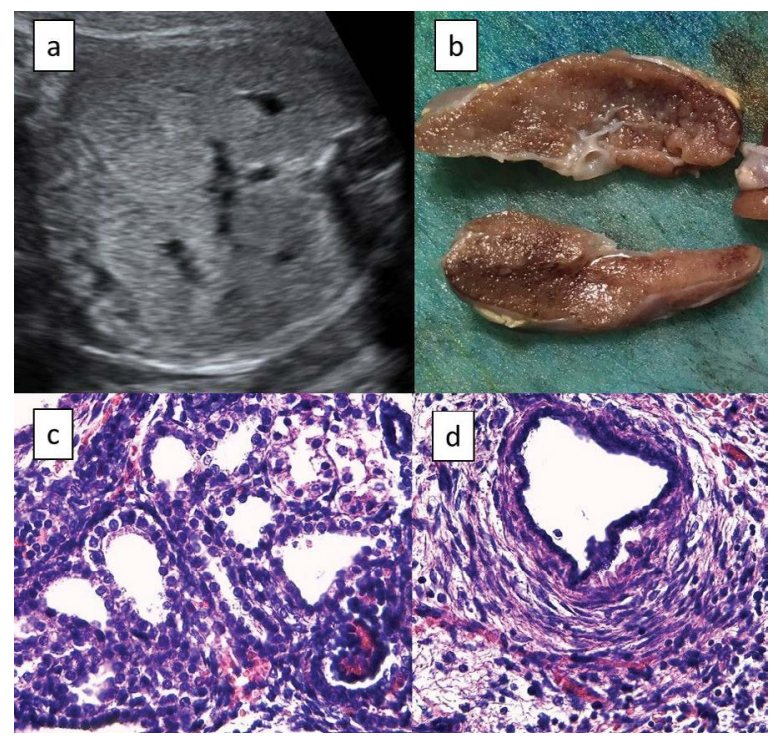

Figure 2. Kidneys in glutaric aciduria type 2; A. Ultrasound imaging at 24 weeks of gestation showing large hyperechoic kidneys; B. Cross section in postmortem examination of 37 weeks newborn - enlarged kidneys without macroscopic cysts; $\mathbf{C}$. Microscopic examination with hematoxylin and eosin staining and magnification $400 \times$ - cortical cysts of the kidney; D. Microscopic examination with hematoxylin and eosin staining and magnification $400 \times$ - medullary cyst of the kidney lary hematopoiesis. Some fibrosis around portal and central spaces of the lobe were detected.

\section{Cytogenetic testing}

Fetal DNA isolated initially from uncultured amniotic fluid and then from cultured amniocytes was obtained. Array comparative hybridization (aCGH) was performed on DNA from uncultured amniotic fluid using CytoSure Constitutional v3 $(8 \times 60$ k) array (Oxford Gene Technology). Karyotype was performed on cultured amniocytes. Karyotype and aCGH revealed normal results for both tests. Parental DNA was isolated from peripheral blood of each parent. Subsequently fetal DNA from cultured amniocytes and parental DNA were sent to the external laboratory for sequencing (CeGaT GmbH, Tübingen, Germany).

\section{Exome sequencing}

ES was performed simultaneously for the fetus, mother and father (trio). SureSelect Human All Exon enrichment kit v.6 (Agilent) was used for library preparation and capture. All laboratory preparations were done according to the manufacturers' protocols. The final products were sequenced on NovaSeq6000 (Illumina) with 100-bp paired-end reads generating raw sequence data stored in FASTQ format. Raw data were post-processed on site using the bcbio-nextgen pipeline [14]. DNA short reads were mapped against human genome reference sequence (GRCh38/hg38) using Burrows-Wheeler Alignment (BWA) and stored as binary Sequence Alignment Map (BAM) [15]. BAM files analysis using Genome Analysis Toolkit (GATK) and variant calling using GATKHaplotype Caller were performed [16, 17]. Next, ANNOVAR was used to annotate relevant information about gene names, predicted variant pathogenicity, reference allele frequencies and metadata from external resources and to add these data to the Variant Call Format (VCF) file [18]. Finally, we used HMZDelFinder algorithm to search for small deletions which were not detected by aCGH [19]. As a control we were able to use exome data from approximately 300 samples sequenced the same platform and processed using the same pipeline. ES analysis revealed novel pathogenic variants on both alleles of ETFDH gene consistent with a diagnosis of GA2. Nonsense variant NM_001281738:C[1191C > A] was of maternal origin and frameshift variant NM_001281738:C[1560A>-] was of paternal origin (Fig. 3). Prenatal ultrasound, prenatal genetic tests and post-mortem examination support the diagnosis of GA2.

\section{RESULTS}

The literature search for prenatal glutaric acidemia yielded a total of 136 publications. Based on metadata and abstracts screening for duplicates and irrelevant publica- 


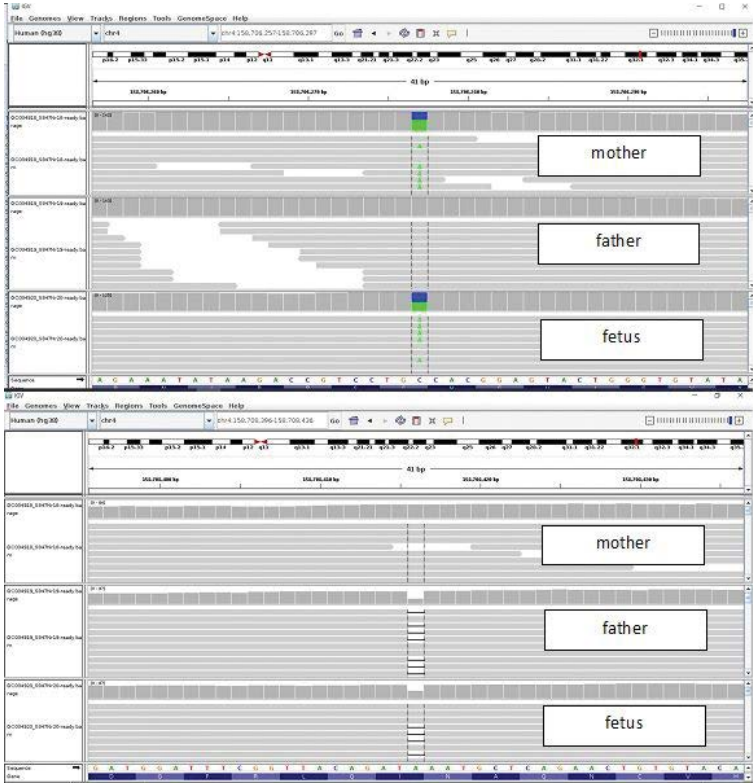

Figure 3. Exome sequencing of trio: nonsense pathogenic variant NM_001281738:C[1191C > A] of ETFDH gene in mother and fetus (upper image), frameshift pathogenic variant NM_001281738:C[1560A>-] of ETFDH gene in father and fetus (lower image). Fetus is compound heterozygote of two pathogenic variants tions were performed and excluded 118 items (e.g. glutaric acidemia type 1). Then, based on full-text analysis nine further publications were excluded for irrelevant content, duplicate cases or no full-text availability (e.g. diagnosis of GA2 in stored material, negative diagnosis of GA2). Nine records were included for final analysis. Eight publications reported a single case and one publication reported two cases. The available data on prenatal diagnosis of GA2 concerning ten cases from literature review and one new case are summarized in Table 1 [20-28].

\section{DISCUSSION}

\section{Prenatal diagnosis of glutaric acidemia 2 \\ - current state of knowledge and clinical implications}

Despite the continuous development of knowledge and technology in the field of genetics and ultrasound diagnostics, a large proportion of cases are still underdiagnosed or undiagnosed. This happens also in the case of $\mathrm{GA} 2$. Although prenatal diagnosis of GA2 is possible, only 10 prenatally diagnosed cases have been reported in over 40 years after first description of the disease [29]. In the ma-

Table 1. Literature review

\begin{tabular}{|c|c|c|c|c|c|}
\hline Ref. & $\begin{array}{l}\text { Family history of } \\
\text { previously diagnosed } \\
\text { GA } 2\end{array}$ & Prenatal presentation & Material & Diagnostic method & Outcome \\
\hline \multirow{2}{*}{20.} & \multirow{2}{*}{ positive } & \multirow{2}{*}{$\mathrm{N} / \mathrm{A}$} & cultured amniotic cells & enzyme activity (RA) & \multirow{2}{*}{ TOP } \\
\hline & & & amniotic fluid & organic acids profile (GC/MS) & \\
\hline \multirow{2}{*}{21.} & \multirow{2}{*}{ positive } & \multirow{2}{*}{ N/A } & cultured amniotic cells & enzyme activity (RA) & \multirow{2}{*}{ TOP } \\
\hline & & & amniotic fluid & organic acids profile (GC/MS) & \\
\hline 22. & positive & $\begin{array}{l}\text { no renal defects in US } \\
\text { raised serum AFP }\end{array}$ & amniotic fluid & organic acids profile (GC/MS) & TOP \\
\hline 23. & positive & N/A & cultured amniotic cells & enzyme activity (RA) & $\begin{array}{l}\text { live born - died } \\
\text { after } 4 \text { months }\end{array}$ \\
\hline \multirow{2}{*}{24.} & \multirow{2}{*}{ positive } & \multirow{2}{*}{ N/A } & cultured amniotic cells & enzyme activity (RA) & \multirow{2}{*}{ TOP } \\
\hline & & & amniotic fluid & organic acids profile (GC/MS) & \\
\hline 25. & positive & no defects in US & cultured amniotic cells & enzyme structure (IMA) & $\begin{array}{l}\text { live born - doing } \\
\text { well at } 6 \text { months }\end{array}$ \\
\hline \multirow{3}{*}{$\begin{array}{l}26 . \\
\text { case } 1\end{array}$} & \multirow{3}{*}{ positive } & \multirow{3}{*}{ N/A } & amniotic fluid & organic acids profile (LC/MS) & \multirow{3}{*}{ TOP } \\
\hline & & & amniotic fluid & acylcarnitine profile (FAB/MS) & \\
\hline & & & maternal urine & acylcarnitine profile (FAB/MS) & \\
\hline \multirow{2}{*}{$\begin{array}{l}26 . \\
\text { case } 2\end{array}$} & \multirow{2}{*}{ positive } & \multirow{2}{*}{$\mathrm{N} / \mathrm{A}$} & amniotic fluid & acylcarnitine profile (FAB/MS) & \multirow{2}{*}{$\begin{array}{l}\text { live born - no } \\
\text { other data }\end{array}$} \\
\hline & & & maternal urine & acylcarnitine profile (FAB/MS) & \\
\hline 27. & positive & $\begin{array}{l}\text { large, hyperechoic kidneys } \\
\text { normal amniotic fluid }\end{array}$ & - & US & TOP \\
\hline \multirow{2}{*}{28.} & \multirow{2}{*}{ positive } & \multirow{2}{*}{$\begin{array}{l}\text { large, hyperechoic kidneys } \\
\text { raised serum and amniotic AFP }\end{array}$} & amniotic fluid & acylcarnitine profile (MS/MS) & \multirow{2}{*}{ TOP } \\
\hline & & & cultured amniotic cells & enzyme activity (SIA) & \\
\hline $\begin{array}{l}\text { present } \\
\text { study }\end{array}$ & negative & $\begin{array}{l}\text { large, hyperechoic kidneys } \\
\text { oligohydramnios }\end{array}$ & cultured amniotic cells & DNA analysis (ES) & $\begin{array}{l}\text { live born - died } \\
\text { after } 2 \text { hours }\end{array}$ \\
\hline
\end{tabular}

US - ultrasound examination; AFP — alpha-fetoprotein; RA - radioisotope assays; GC/MS — gas chromatography/mass spectrometry; IMA — immunochemical assays; LC/MS — liquid chromatography/mass spectrometry; FAB/MS — fast atom bombardment/ mass spectrometry; MS/MS — tandem mass spectrometry; SIA — stable isotope assays; $\mathrm{ES}$ — exome sequencing; TOP — termination of pregnancy; N/A — not available 
jority of cases prenatal diagnosis relied on enzyme activity measurement in cultured amniotic cells and/or glutaric acid measurement in the amniotic fluid while in other cases it was based on acylcarnitine profile in maternal urine and/or amniotic fluid, enzyme structure analysis or on ultrasound examination [20-28]. Measurement of enzyme activity is time-consuming, troublesome and available in a limited number of laboratories [30]. On the other hand glutaric acid measurement in the amniotic fluid by gas or liquid chromatography/mass spectrometry (GC/MS or LC/MS) is fast and simple, but it is prone to false negative diagnosis if the fetus does not excrete large amount of glutaric acid [26, 31]. Both tests are frequently used simultaneously to minimize the risk of misdiagnosis. Furthermore, acylcarnitine profile in the maternal urine may be unreliable as both abnormal as well as normal values have been reported in affected fetuses $[26,32]$. DNA sequencing targeted for known pathogenic variants of ETFA, ETFB and ETFDH genes has already been performed in fetuses at risk of GA2. It demonstrated its potential usefulness as a diagnostic tool by giving negative results in unaffected fetuses [7]. Molecular analysis has also an advantage in cases with severe oligohydramnios as DNA can be isolated from fetal blood or trophoblast tissue not only from amniotic fluid [33]. However, it should be emphasized that all these methods were useful in cases with a positive history of GA2 running in the family. In all cases, history of death or severe illness of the previous child due to confirmed GA2 pointed out targeted prenatal diagnosis in the next pregnancy [20, 21, 23-26]. In three cases prenatal manifestation was observed in previous pregnancies as enlarged hyperechoic or cystic kidneys and elevated serum alfa fetoprotein (AFP) level but they were diagnosed as GA2 in postmortem examinations. In these three cases renal anomalies reoccur in the next pregnancy leading to the exact diagnosis $[22,27,28]$. In our study trio analysis was performed as it significantly improves the diagnostic yield compared with proband-only testing [34]. Consequently, we were able to identify two novel pathogenic variants of the ETFDH gene and to indicate their parental origin. These results allow the establishment of correct diagnosis in the affected fetus and calculate genetic risk in the family which meets expectations among Polish women regarding prenatal diagnosis [35]. Moreover, identification of novel variants enriches existing databases of single nucleotide polymorphism.

\section{SUMMARY}

Inborn errors of metabolism are rare disorders with unspecific manifestation in prenatal settings. Hyperechoic, enlarged kidneys could be one of the clinical features of metabolic diseases. After exclusion of chromosomal abnormalities, urinary tract obstruction and intrauterine infec- tions, glutaric acidemia type 2 and number of monogenic disorders should be considered. Exome sequencing approach allows to identify pathogenic variants even without earlier knowledge of the precise genetic background. This strategy could help in early diagnosis, optimal perinatal care and family planning for affected individuals.

\section{Conflict of interest}

All authors declare no conflict of interest.

\section{Acknowledgments}

Funding sources: subsidy from the state budget for the Institute of Mother and Child for 2019 to BN

\section{REFERENCES}

1. Arnold GL. Inborn errors of metabolism in the 21 century: past to present. Ann Transl Med. 2018;6(24): 467, doi: 10.21037/atm.2018.11.36, indexed in Pubmed: 30740398.

2. Henriques BJ, Bross $P$, Gomes CM. Mutational hotspots in electron transfer flavoprotein underlie defective folding and function in multiple acyl-CoA dehydrogenase deficiency. Biochim Biophys Acta. 2010; 1802(11): 1070-1077, doi: 10.1016/j.bbadis.2010.07.015, indexed in Pubmed: 20674745.

3. Olsen RKJ, Andresen BS, Christensen E, et al. Clear relationship between ETF/ETFDH genotype and phenotype in patients with multiple acyl-CoA dehydrogenation deficiency. Hum Mutat. 2003; 22(1): 12-23, doi: 10.1002/humu.10226, indexed in Pubmed: 12815589.

4. Taniguchi K, Kawai T, Hata K. Placental Development and Nutritional Environment. Adv Exp Med Biol. 2018; 1012: 63-73, doi: 10.1007/978981-10-5526-3 7, indexed in Pubmed: 29956195.

5. Guibaud L, Collardeau-Frachon S, Lacalm A, et al. Antenatal manifestations of inborn errors of metabolism: prenatal imaging findings. J Inherit Metab Dis. 2017; 40(1): 103-112, doi: 10.1007/s10545-016-9992-3, indexed in Pubmed: 27853988.

6. Schulze $A$, Lindner $M$, Kohlmüller $D$, et al. Expanded newborn screening for inborn errors of metabolism by electrospray ionization-tandem mass spectrometry: results, outcome, and implications. Pediatrics. 2003; 111 (6 Pt 1): 1399-1406, doi: 10.1542/peds.111.6.1399, indexed in Pubmed: 12777559.

7. Olsen RKJ, Andresen BS, Christensen E, et al. DNA-based prenatal diagnosis for severe and variant forms of multiple acyl-CoA dehydrogenation deficiency. Prenat Diagn. 2005; 25(1): 60-64, doi: 10.1002/pd.983, indexed in Pubmed: 15662686.

8. Lehnert W, Wendel U, Lindenmaier S, et al. Multiple acyl-CoA dehydrogenation deficiency (glutaric aciduria type II), congenital polycystic kidneys, and symmetric warty dysplasia of the cerebral cortex in two brothers. I. Clinical, metabolical, and biochemical findings. Eur J Pediatr. 1982; 139(1): 56-59, doi: 10.1007/BF00442081, indexed in Pubmed: 7173259.

9. Frerman FE, Goodman SI. Defects of electron transfer flavoprotein and electron transfer flavoprotein-ubiquinone oxidoreductase: glutaric acidemia type II. In: Scriver CR, Beaudet AL, Sly WS, Valle D. ed. The Metabolic and Molecular Bases of Inherited Disease. 8th ed. McGraw-Hill Publishers, New York 2001.

10. Indo Y, Glassberg R, Yokota I, et al. Molecular characterization of variant alpha-subunit of electron transfer flavoprotein in three patients with glutaric acidemia type II--and identification of glycine substitution for valine-157 in the sequence of the precursor, producing an unstable mature protein in a patient. Am J Hum Genet. 1991; 49(3): 575-580, indexed in Pubmed: 1882842.

11. Colombo I, Finocchiaro G, Garavaglia B, et al. Mutations and polymorphisms of the gene encoding the beta-subunit of the electron transfer flavoprotein in three patients with glutaric acidemia type II. Hum Mol Genet. 1994; 3(3): 429-435, doi: 10.1093/hmg/3.3.429, indexed in Pubmed: 7912128.

12. Beard SE, Spector EB, Seltzer WK, et al. Mutations in electron transfer flavoprotein: ubiquinone oxidoreductase (ETF:QO) in glutaric acidemia type II. Clin Res. 1993; 41: 271A. 
13. Avni FE, Garel $\mathrm{C}$, Cassart $\mathrm{M}$, et al. Imaging and classification of congenital cystic renal diseases. AJR Am J Roentgenol. 2012; 198(5): 1004-1013, doi: 10.2214/AJR.11.8083, indexed in Pubmed: 22528889.

14. https://github.com/bcbio/bcbio-nextgen.

15. Li H, Durbin R, Li H, et al. Fast and accurate short read alignment with Burrows-Wheeler transform. Bioinformatics. 2009; 25(14): 1754-1760, doi: 10.1093/bioinformatics/btp324, indexed in Pubmed: 19451168.

16. Poplin R, Ruano-Rubio V, DePristo $M$, et al. Scaling accurate genetic variant discovery to tens of thousands of samples. , doi: 10.1101/201178.

17. McKenna A, Hanna M, Banks E, et al. The Genome Analysis Toolkit: a MapReduce framework for analyzing next-generation DNA sequencing data. Genome Res. 2010; 20(9): 1297-1303, doi: 10.1101/gr.107524.110, indexed in Pubmed: 20644199.

18. Wang K, Li M, Hakonarson H. ANNOVAR: functional annotation of genetic variants from high-throughput sequencing data. Nucleic Acids Res. 2010; 38(16): e164, doi: 10.1093/nar/gkq603, indexed in Pubmed: 20601685.

19. Gambin T, Akdemir ZC, Yuan Bo, et al. Homozygous and hemizygous CNV detection from exome sequencing data in a Mendelian disease cohort. Nucleic Acids Res. 2017; 45(4): 1633-1648, doi: 10.1093/nar/gkw1237, indexed in Pubmed: 27980096.

20. Goodman SI, Gallegos DA, Pullin CJ, et al. Antenatal diagnosis of glutaric acidemia. Am J Hum Genet. 1980; 32(5): 695-699, indexed in Pubmed: 6893520.

21. Mitchell G, Saudubray JM, Benoit Y, et al. Antenatal diagnosis of glutaric aciduria type II. Lancet. 1983: 1099.

22. Boué J, Chalmers RA, Tracey BM, et al. Prenatal diagnosis of dysmorphic neonatal-lethal type II glutaric aciduria. Lancet. 1984: 846-847.

23. Bennett MJ, Curnock DA, Engel PC, et al. Glutaric aciduria type II: biochemical investigation and treatment of a child diagnosed prenatally. J Inherit Metab Dis. 1984; 7(2): 57-61, doi: 10.1007/BF01805802, indexed in Pubmed: 6207379.

24. Henderson HE, Balla R, de Jo, et al. Postnatal and antenatal laboratory diagnosis of glutaric aciduria II in a South African family. S Afr Med J. 1987; 71: 589-591.

25. Yamaguchi S, Shimizu N, Orii T, et al. Prenatal diagnosis and neonatal monitoring of a fetus with glutaric aciduria type II due to electron transfer flavoprotein (beta-subunit) deficiency. Pediatr Res. 1991;
30(5): 439-443, doi: 10.1203/00006450-199111000-00009, indexed in Pubmed: 1754299.

26. Sakuma T, Sugiyama N, Ichiki T, et al. Analysis of acylcarnitines in maternal urine for prenatal diagnosis of glutaric aciduria type 2. Prenat Diagn. 1991; 11(2): 77-82, doi: 10.1002/pd.1970110203, indexed in Pubmed: 2062823.

27. Kjaergaard S, Graem N, Larsen T, et al. Recurrent fetal polycystic kidneys associated with glutaric aciduria type II. APMIS. 1998; 106(12): 1188-1193, doi: 10.1111/j.1699-0463.1998.tb00276.x, indexed in Pubmed: 10052728.

28. Chisholm CA, Vavelidis F, Lovell MA, et al. Prenatal diagnosis of multiple acyl-CoA dehydrogenase deficiency: association with elevated alpha-fetoprotein and cystic renal changes. Prenat Diagn. 2001; 21(10): 856-859, doi: 10.1002/pd.157, indexed in Pubmed: 11746129.

29. Przyrembel H, Wendel U, Becker K, et al. Glutaric aciduria type II: report on a previously undescribed metabolic disorder. Clin Chim Acta. 1976; 66(2): 227-239, doi: 10.1016/0009-8981(76)90060-7, indexed in Pubmed: 1245071.

30. https://www.ncbi.nlm.nih.gov/gtr/conditions/C0268596/.

31. Goodman SI. Prenatal diagnosis of glutaric acidemias. Prenat Diagn. 2001; 21(13): 1167-1168, doi: 10.1002/pd.201, indexed in Pubmed: 11787045.

32. Manning NJ, Bonham JR, Downing $M$, et al. Normal acylcarnitines in maternal urine during a pregnancy affected by glutaric aciduria type II. J Inherit Metab Dis. 1999; 22(1): 88-89, doi: 10.1023/a:1005467803802, indexed in Pubmed: 10070625.

33. Łaczmańska I, Stembalska A. [New molecular methods in prenatal invasive diagnostics]. Ginekol Pol. 2013; 84(10): 871-876, indexed in Pubmed: 24273910.

34. Yates CL, Monaghan KG, Copenheaver D, et al. Whole-exome sequencing on deceased fetuses with ultrasound anomalies: expanding our knowledge of genetic disease during fetal development. Genet Med. 2017; 19(10): 1171-1178, doi: 10.1038/gim.2017.31, indexed in Pubmed: 28425981.

35. Kosinski P, Ferreira JC, Lipa M, et al. Preferences and expectations among Polish women regarding prenatal screening. Ginekol Pol. 2019; 90(9): 544-548, doi: 10.5603/GP.2019.0094, indexed in Pubmed: 31588553. 